
KAMIL TORRES ${ }^{1}$, ANNA TORRES $^{1}$, RYSZARD MACIEJEWSKI $^{1}$

\title{
Evaluation of patients' satisfaction and quality of life in selected healthcare institutions in southern-eastern Poland
}

\begin{abstract}
Introduction. In recent times, patient outcome measurement has developed from being narrowly focussed upon levels of symptomatology and service use, to being a broader assessment of the impact of illness and treatment on the individual. Thus, it can be said that quality of life has become as significant as life expectance. This has brought about a transition in the assessment of treatment. Quality of life (QOL) is a multidimensional concept that usually includes subjective evaluations of both positive and negative aspects of life as it is being led. With regard to healthcare, a cross-sectional comparison of palliative care needs is crucial in understanding differences in the patients' quality of life. Hence, an analysis of programme implementation within different types of healthcare institutions is significant in evaluating current medical care standards. Our study analyzed the satisfaction level and quality of life of patients with Chronic Obstructive Pulmonary Disease (COPD), and after myocardial infarction (MI). Different types of healthcare institutions were evaluated.

Aim. To evaluate patients' satisfaction and quality of life in selected healthcare institutions in southern-eastern Poland.

Material and methods. The quality of life of patients with Chronic Obstructive Pulmonary Disease (COPD) was analyzed through the medical documentation obtained from different types of healthcare institutions. Among these are the pulmonary outpatient department in Moczary, as well as a GP Practice and a Nursing Home in this location. The quality of life of patients after myocardial infraction was analyzed through a survey study conducted at "Polonia" hospital spa in Rymanów Zdrój (the cardiology department).

Results and conclusions. The post-myocardial infarction incident patients had began to care more about their health condition and the quality of life they led. Moreover, their satisfaction level from received treatment and medical care indicated that the cardiological services implemented in southern-eastern Poland has proceeded in a good direction. Of note, these patients were systematically under specialist control. In contrast, among patients with COPD, only those under everyday care in the Nursing Home in Moczary received a similar systematic treatment, thus, COPD patients in Moczary lead a poor quality of life. This indicates a need to re-evaluate the current programmes and services provided by health care institutions in this region.
\end{abstract}

Keywords: Myocardial Infarction, Chronic Obstructive Pulmonary Disease, Quality of life.

DOI: $10.1515 /$ pjph-2015-0004

\section{INTRODUCTION}

Quality of life (QOL) is a broad multidimensional concept that usually includes subjective evaluations of both positive and negative aspects of life. Chronic Obstructive Pulmonary Disease (COPD) and cardiovascular events such as myocardial infarction (MI) are common in the general population, and they place a considerable burden on patients. This disease has a negative effect upon the quality of a patient's life, and QOL in patients with COPD is affected by many factors. In addition to spirometric abnormalities, significant factors modifying QOL are educational background, monthly income, present occupation and employment status [1]. Patients with coronary heart disease also often suffer from an impaired health-related quality of life (HRQoL). A healthier lifestyle not only extends individuals' life span, but might also improve their HRQoL, and better HRQoL scores were found in those coronary patients who adopted a healthier lifestyle [2]. Quality of life, in recent years, has been the subject of special interest and of many studies. It also has begun to be seen as being as significant as life expectance. This has brought about a transition in the assessment of treatment effects and a greater consciousness of having patients actively involved in the medicinal process. Hence, a cross-sectional comparison of healthcare needs is crucial in understanding differences in patients' quality of life.

\footnotetext{
${ }^{1}$ Laboratory of Medical Simulation, Department of Human Anatomy, Medical University of Lublin, Poland

${ }^{2}$ Students' Scientific Association, Department of Public Health, University of Information Technology and Management, Rzeszów, Poland
} 


\section{AIM}

The aim of the present study was to evaluate patients' satisfaction and quality of life as experienced through their contact with, and after treatment in selected healthcare institutions in southern-eastern Poland.

\section{MATERIAL AND METHODS}

The quality of life of patients with Chronic Obstructive Pulmonary Disease (COPD) was analyzed through medical documentation obtained from the pulmonary outpatient department, a GP Practice and a nursing home in Moczary. This analysis was based on the medical records of patients suffering from COPD, and the studied population ranged in age from 50 to 100 years. The COPD patients' quality of life study involved 86 patients (11 women $-23 \%$ and 37 men - 77\%) diagnosed with Chronic Obstructive Pulmonary Disease. Of these individuals, $82 \%$ of respondents came from the rural area, while only $18 \%$ of all patients came from the urban environment. All participants in the study were placed within three groups. The first group consisted of patients under GP control and who experienced non-systematic treatment. The second group was composed of pulmonary outpatient department patients. The last, (the third group) consisted of patients who have developed COPD, and were under the everyday care of the nursing home in Moczary.

The quality of life of patients after myocardial infraction (MI) was analyzed through a survey study. This study was conducted at "Polonia" hospital spa in Rymanów Zdrój, within the cardiology department. It involved 58 patients (31 women $-53 \%$ and 27 men $47 \%$ ) after MI. The patients were between 31-82 years. Part of the studied group came from the rural area $(34.59 \%$ ), while $24.41 \%$ of all patients came from the urban environment. The survey study included questions involving the following topics: basic information about the occurrence of myocardial infarction and its possible causes, health condition before and after incident, feelings associated with current condition and patients' opinions about received rehabilitation.

\section{RESULTS}

Our study showed that the group of patients after myocardial infraction was more involved in systematic treatment and health controls (especially after the incident) than were a group with diagnosed COPD. Indeed, $56 \%$ of all patients within the study with diagnosed COPD were not taking regular treatment, in most cases, due to lack of funds for the purchase of inhaled drugs and for meeting pulmonary clinic visit costs. From the analyzed medical records, it is clear that almost all respondents worked physically in the woods or were living on small farms. Moreover, $18 \%$ of the patients from that group were under the care of the local Social Welfare Centre. What is more, $26 \%$ of these patients were under specialist control. Furthermore, ten patients who had been referred to the specialist clinic did not accept a referral from their family doctor, due to the lack of money for specialist doctor visits and for meeting possible drug prescription costs. In addition, a limited number of buses connecting the specialist clinic and their homes was another reason that was given.

The group of patients after MI was more able to systematically attempt to follow specialist controls before and after the incident. Indeed, the study on the group of patients after MI showed that after the incident, patients began to care more about their health and to systematically control their health condition. Before their MI incident, blood pressure was regularly controlled by $41 \%$ of the patients, and after MI, by $67 \%$ of the patients. In addition, blood sugar levels were checked by $28 \%$ of the patients before MI, and $34 \%$ after MI, while cholesterol levels was controlled systematically in $21 \%$ of the patients before MI, and $31 \%$ of the patients after MI. All told, the percentage of the patients that controlled their health increased from $22 \%$ before MI, to $72 \%$ after MI. Furthermore, the analysis of patients problems that emerged after myocardial infarction showed that the most common among them is fast fatigue (23\%) and flushing (19\%). The least number of patients complain of dizziness $(5 \%)$ and diabetes (1\%). Results are presented in Table 1.

TABLE 1. Problems that emerged after myocardial infarction.

\begin{tabular}{cc}
\hline TYPE OF PROBLEMS & $\mathbf{\%}$ \\
\hline Fast fatigue & 23 \\
\hline Flushing & 19 \\
\hline Hypertension & 18 \\
\hline Increase in blood pressure & 15 \\
\hline Shortness of breath & 13 \\
\hline Decrease in blood pressure & 6 \\
\hline Dizziness & 5 \\
\hline Diabetes & 1
\end{tabular}

What is more, patients with the risk of further cardiovascular incidents were systematically under specialist care, who noted that they were exposed to high stress levels. Analysis of feelings of the patients after myocardial infarction revealed that the most common type of feelings experienced by those patients is general fear $(19 \%)$. The second most common type of feelings is fear of themselves and their live $(18 \%)$. The smallest group of the patients experienced fear of physical limitations (4\%) and social acceptance fear (3\%). Results are presented in Table 2.

TABLE 2. Feelings associated patients after MI.

\begin{tabular}{cc}
\hline \hline TYPE OF FEELINGS & $\mathbf{\%}$ \\
\hline General fear & 19 \\
\hline Fear of themselves and their live & 18 \\
\hline Fear of relatives & 16 \\
\hline Schock & 11 \\
\hline Feeling of guilty & 11 \\
\hline Fear of job lost & 9 \\
\hline Fear of losing physical atraction & 5 \\
\hline Physical limitations fear & 4 \\
\hline Social acceptance fear & 3
\end{tabular}




\section{DISCUSSION}

The term 'Specialist Healthcare' refers to the comprehensive management of the physical, psychological, social, spiritual and existential needs of patients, in particular, those with incurable, progressive illnesses. The goal of specialist care is to achieve within patients, the best possible quality of life through relief of suffering, the control of symptoms and the restoration of functional capacity while remaining sensitive to personal, cultural and religious values, beliefs and practices [3]. Chronic Obstructive Pulmonary Disease (COPD) and myocardial infarction are common diseases within the Polish population [4,5]. Both are progressive and their exacerbations are a threat to life. The appropriate pharmacotherapy slows the progression of these diseases, reduces the number of exacerbations and improves quality of life $[5,6]$. Due to the specificity of the diseases, these groups of patients demand specialist and continuous health care. Thus, the study ascertained how patients assessed the impact of health services on their quality of life. In undertaking this evaluation, their quality of life was analyzed together with the intensity of care encountered at different types of healthcare institutions in southern-eastern Poland. This last term - 'Intensity of Care' refers to the amount of care provided at services and health institutions, and it is comprised of duration and/or frequency of interventions - the domain of content of care [7]. Quality of life measurement is an important aspect of healthcare, given that maximizing this item is the main aim of this type of care. A large variety of quality of life measurement instruments are appropriate for use in COPD and MI, but, unfortunately, most have not been adequately evaluated so the effectiveness of the pharmacological and non-pharmacological interventions is not always easy to assess [8].

In practice, patients with COPD generally sought medical attention because of the symptoms they endured, particularly breathlessness and the resulting physical limitations. These affected their health-related quality of life (HR-QOL). The defining feature of COPD is an airflow limitation that causes air trapping and increases hyperinflation, as the ventilation rate is enhanced during physical effort. Hyperinflation causes or worsens breathlessness as breathing becomes inefficient, with the end result being an avoidance of physical exertion and a cycle of increasing dyspnea caused by inactivity and de-conditioning, with resulting deleterious effects on HR-QOL. Current published guidelines for COPD state that the goals of pharmacologic therapy should be to control symptoms, as well as to improve health status and exercise tolerance [9].

Patients diagnosed with COPD who were staying in the Nursing Home, were under regular pulmonary outpatient department control and received systematical treatment. This group of patients was able to be treated continuously because they did not have to arrange visits, purchase medicine and could access the specialist pulmonary clinic. Being a patient of the Nursing Home was, thus, a guarantee of realizing proper care. The analysis of the data showed that almost all such patients struggled with alcoholism, a difficult financial situation, homelessness and a lack of family support. Without the assistance of the Nursing Home, these COPD patients would have had to be included within the group encountering non-systematic treatment. Long term untreated COPD, difficult living conditions and alcohol lead to a set of diverse kinds of diseases and ailments that coexist with COPD. This situation greatly hinders patients' life style practices.

Through the data analysis, it can be concluded that the continuous nature of the disease constrains patients' activity in terms of the physical, emotional and social spheres, hence, dramatically lowering their quality of life. Therefore, the depression and anxiety, which, in some cases, accompanied COPD, adversely affected the health of the patient and increased the number of hospitalizations. Our study shows that severe symptoms of depression and anxiety correlate both with poor prognosis and reduced quality of life of patients with COPD. In the group of patients with coexisting depression, a reduced tendency for the use of medical recommendations, as well as a reduced intake of prescribed medication were observed, which, in turn, led to exacerbation of the underlying disease. From the analysis of the collected medical data, our work reveals that $6 \%$ of all patients participating in the study suffered from and were treated for depression. Furthermore, our study reveals that as a result of myocardial infarction, those affected, experience different feelings and fears. Indeed, our survey study shows that most frequently, respondents experienced general feelings of fear $(19 \%)$, fear of themselves and their lives (18\%) and fear of relatives $(16 \%)$.

The survey we undertook asked patients to list the problems that emerged after MI. These patients most commonly pointed out rapid fatigue (23\%), hypertension (18\%) and flushing (19\%). Patients after MI were also given a second survey. This included questions about their opinion towards the rehabilitation quality that they received in the Rymanów Zdrój hospital spa. Here, physiotherapists play an inherent role in the multidisciplinary palliative care team, and emphasis is placed upon improving the function and quality of life in patients who are deemed to require both physical and functional dimensions of care [10]. The results of our survey show that $40 \%$ of the patients described receiving rehabilitation that they conceive as being very good, and, for $60 \%$, good.

What is more, there was no negative assessment of received rehabilitation among patients. Moreover, 94\% of those surveyed felt mental comfort during rehabilitation, however, $6 \%$ of the patients stated otherwise. During their stay in the "Polonia" hospital spa in Rymanów Zdrój, patients received various physiotherapy interventions. The most common of these included kinesiotherapy, electrical and thermal modalities, and actinotherapy (including laser and magnetic field therapies).

\section{CONCLUSIONS}

1. The group of patients who experienced an MI incident began to care more about their health condition and quality of life. Moreover, they enjoyed systematic specialist control. In contrast, of the group with COPD, only one group of patients, those staying at the Nursing Home in Moczary, experienced systematic treatment. 
2. The quality of life of the studied group of patients after MI, and their satisfaction with regard to the treatment received while in the cardiology department in the "Polonia" hospital spa, is better than that of the patients with COPD treated by way of the pulmonary outpatient department and GP practice in Moczary.

3. The patients after MI found that the healthcare system they experienced is more effective than that of the healthcare system of patients with COPD.

4. In both study groups, patients felt their quality of life constrained by negative feelings and fears.

\section{REFERENCES}

1. Bak-Drabik K, Ziora D. The impact of socioeconomic status on the quality of life in patients with chronic obstructive pulmonary disease. Pneumonol Alergol Pol. 2010;78(1):3-13

2. Smedt DD, Clays E, Annemans L, et al. The association between selfreported lifestyle changes and health-related quality of life in coronary patients: the EUROASPIRE III survey. Eur J Prev Cardiol. 2013 10;21(7):796-805.

3. Last Acts Task Force. Robert Wood Johnson Foundation: Precepts of Palliative Care. Princeton, NJ;1997.

4. Bednarek M, Maciejewski J, Woźniak M, et al. Prevalence, severity and under diagnosis of COPD in the primary care setting. Thorax. 2008;63:402-7.

5. Bandosz P, O'Flaherty M, Drygas W, et al. Decline in mortality from coronary heart disease in Poland after socioeconomic transformation: a modeling study. BMJ. 2012;344:d8136.

6. Global Strategy for the Diagnosis, Management and Prevention of COPD. Global Initiative for Chronic Obstructive Lung Disease (GOLD). Updated 2013.

7. Brynmor Lloyd-Evans, Acute inpatient mental health wards and inpatient alternatives: a quantitative comparison of the care provided, UCL,PhD Thesis

8. Albers G, Echteld MA, de Vet HC, et al. Evaluation of quality-of-life measures for use in palliative care: a systematic review. Palliat Med. 2010;24:17-37.

9. Feldman GJ. Improving the quality of life in patients with chronic obstructive pulmonary disease: focus on indacaterol. Int J Chron Obstruct Pulmon Dis. 2013;8:89-96.

10. Egan KA, Abbott P. Interdisciplinary team training - preparing new employees for the specialty of hospice and palliative care. J Hosp Palliat Nurs. 2002;4:161-71.

\section{Corresponding author}

Ewa Domańska-Glonek, M.D.

Laboratory of Medical Simulation, Department of Human Anatomy,

Medical University of Lublin

4 Jaczewskiego Str., 20-094 Lublin

tel. +48814486050 ;

E-mail: ewa_glonek@op.pl 